\title{
Presence of Candida spp. in the oral cavity of heart transplantation patients
}

\author{
Patrícia Monteiro RIBEIRO', Fernando BACAL ${ }^{2}$, Cristiane Yumi KOGA-ITO ${ }^{3}$, Juliana Campos JUNQUEIRA ${ }^{3}$, \\ Antonio Olavo Cardoso JORGE ${ }^{3}$ \\ 1- PhD, Department of Bioscience and Oral Diagnosis, São José dos Campos Dental School, São Paulo State University, São José dos Campos, SP, Brazil. \\ 2- PhD, Heart Institute (INCOR/HCFMUSP), Medical School, University of São Paulo, São Paulo, SP, Brazil. \\ 3- PhD, Professor of Microbiology and Immunology, Department of Bioscience and Oral Diagnosis, São José dos Campos Dental School, São Paulo State \\ University, São José dos Campos, SP, Brazil.
}

Corresponding address: Antonio Olavo Cardoso Jorge - Faculdade de Odontologia de São José dos Campos - UNESP - Departamento de Biociências e Diagnóstico Bucal - Disciplina de Microbiologia e Imunologia - Av. Francisco José Longo, 777, São José dos Campos - SP - 12.245-000 - Brasil - Phone: 055 1239479033 - Fax: 0551239479010 - e-mail: olavojorge@fosjc.unesp.br

Received: May 28, 2009 - Modification: April 27, 2010 - Accepted: May 21, 2010

\section{ABSTRACT}

\begin{abstract}
Candida spp. can lead to infections or even fungal sepsis particularly among immunocompromized individuals. Objective: The aim of the present study was to analyze the presence of Candida spp. among patients subjected to orthotopic heart transplantation. Material and Methods: Oral rinses from 50 patients subjected to orthotopic heart transplantation, aged 13 to 70 years, 40 males and 10 females, were examined. Sexage-oral conditions matched-control included 50 individuals who were not subjected to any kind of transplantation and were not immunocompromized for any other reason. Counts of yeasts were expressed as median values of logarithm of cfu/mL and were statistically compared by Mann-Whitney's test. The heart transplant and control groups were compared for the presence of Candida spp. by chi-square test $(p<0.05)$. Results: The results showed statistically significant difference $(p=0.001)$ in the prevalence of Candida spp. between the transplantation and control groups. Counts of yeasts (cfu/mL) in the transplanted group were significantly higher than in the control group $(p=0.005)$. Candida albicans was the most prevalent species isolated from both groups. Conclusions: It was concluded that Candida yeast counts were higher in the heart transplant recipients than in the controls. There was higher variation of Candida species among the heart transplant patients and the most frequently isolated samples were: Candida albicans, Candida glabrata and Candida tropicalis. Isolates of Candida dubliniensis was not found in either of the groups.
\end{abstract}

Key words: Candida. Heart transplantation. Oral. Fungal infections.

\section{INTRODUCTION}

Candidiasis occurs in organ transplanted patients that are in immunosuppression treatment and carriers of acquired immunodeficiency virus $2,3,5,8,26,30$. If disseminated, these infections are related to high morbidity and mortality levels ${ }^{1,20}$. Candida albicans is the etiological agent of most of oropharyngeal candidiasis cases associated with immunosuppression, although infections caused by $C$. tropicalis and $C$. dubliniensis have also been reported $^{13,21}$.

A heart transplant patient undergoing immunosuppressive therapy receives cyclosporine, azathioprine and prednisone or tacrolimus (FK506), and rapamycin, among others ${ }^{10,26}$. The main causes of death in the immediate period, up to the first year following heart transplantation, are infection and rejection ${ }^{6,26}$. The incidence of fungal infection in patients receiving a heart transplant varies between $10 \%$ and $25 \%$. Extreme cases have also been reported, where the cause of death was fungal sepsis ${ }^{5,6}$. The levels of Candida spp. in the oral cavity of heart transplant recipients can lead to a better understanding of the relationship among host, immunosuppression and the fungus development, improving the quality of life of these patients. The present study evaluated the prevalence of Candida spp. in the oral cavity of orthotopic heart transplant recipients. 


\section{MATERIAL AND METHODS}

\section{Ethic Aspects}

This study was approved by the Research Ethics Committee of the São José dos Campos Dental School/UNESP (060/2001 PH/CEP) and the Medical School of the University of São Paulo/INCOR/ HCFMUSP (1004/01).

\section{Subjects}

\section{Inclusion criteria}

One-hundred individuals were included in this study. Fifty patients were recipients of orthotopic heart transplantation, aged 13 to 70 years (mean age $=48.6$ years), 40 males $(80 \%)$ and 10 females (20\%). These patients received immunosuppressive therapy for a minimum period of 2 months and were under clinical follow-up at Univeristy of São Paulo Medical School's Heart Institute (INCOR/HCFMUSP).

Sex-age-oral conditions matched control group included 50 individuals who were not subjected to any kind of organ transplantation. These individuals were aged 13 to 70 years (mean age $=47.5$ years), 40 males $(80 \%)$ and 10 females $(20 \%)$, with the closest oral conditions to those of the heart transplant patients (regarding the use of denture or orthodontic appliances). These individuals were under treatment at São José dos Campos Dental School, São Paulo State University, Brazi.

\section{Exclusion criteria}

Patients who presented clinical symptoms of oral candidiasis or were under antifungal or antibacterial therapy were excluded from the study. Patients under any kind of immunosuppressive therapy were excluded from the control group.

\section{Oral Rinse Collection}

Each individual received $10 \mathrm{~mL}$ of sterile phosphate-buffered saline (PBS; $0.1 \mathrm{M}$ and $\mathrm{pH}$ 7.2) supplied in a disposable universal sterile container. They were required to mouthwash for 30 $s$ and collect the oral rinse back into the previously identified container. This container was kept in ice in a thermal bag, and was forwarded to the microbiology laboratory within a maximum time span of $3 \mathrm{~h}$ between sampling and processing. Each sample was centrifuged at 2,300 xg for 10 min (Centrifuge MPW-350R; Biosystems, São José dos Pinhais, PR, Brazil) and the supernatant was discharged. Then, the deposit was resuspended in $2.5 \mathrm{~mL}$ of PBS and mixed in a vortex equipment (Phoenix, model AP56, Araraquara, SP, Brazil) for $30 \mathrm{~s}$, thus producing the final concentration suspension.

\section{Collection of Yeast Isolates}

An amount of $0.1 \mathrm{~mL}$ from all obtained suspensions was cultured in duplicate on Sabouraud dextrose agar (Difco Laboratories, Detroit, MI, USA) with chloramphenicol $(0.1 \mathrm{mg} / \mathrm{mL}$ of culture medium) and also in duplicate on CHROMagar (CHROMagar; Microbiology, Paris, France). Plates were incubated at $37^{\circ} \mathrm{C}$ for $48 \mathrm{~h}$ and kept at room temperature for the following 5 days. After, the number of colonyforming unities per mililiter (cfu/mL) of yeasts was calculated. Stained smears were obtained by Gram method for microscopic confirmation and yeast cfu were transferred to Sabouraud dextrose agar, so as to obtain pure cultures that were later identified. All cfu that showed different color or morphology in CHROMagar were isolated.

\section{Candida Species Identification}

Initial presumptive identification was based on colonies morphology and colour observed in CHROMagar. Isolates were phenotypically identified according to Samaranayake and Macfarlane ${ }^{24}$ (1990) and Sandvén ${ }^{25}$ (1990). The following tests were performed: germ-tube production, chlamidoconidia, hyphae/pseudohyphae production on Corn Meal agar (Difco), carbohydrate fermentation and assimilation. Orange-red colonies with Rhodotorula spp. features were confirmed by urease testing.

\section{Genotipic Identification of $\boldsymbol{C}$. dubliniensis (Polymerase Chain Reaction - PCR)}

Isolates identified phenotypically as $C$. albicans or C. dubliniensis were subjected to molecular detection of $C$. dubliniensis. These isolates were analyzed by PCR, according to the methodology proposed by Donnelly, et al.7 (1999) and Mähnb, et al. ${ }^{16}$ (2005), with modifications. Briefly, the isolates were plated on Sabouraud dextrose agar and incubated for $24 \mathrm{~h}$ at $37^{\circ} \mathrm{C}$. Then, a single colony was transferred to $75 \mathrm{~mL}$ of zymoliase $(0.5 \mathrm{mg} / \mathrm{mL})$ (Sigma, St. Louis, MO, USA) solution $(0.5 \mathrm{mg} / \mathrm{mL}$ in $1 \mathrm{M}$ sorbitol buffer). Tubes were maintained at $95^{\circ} \mathrm{C}$ for $10 \mathrm{~min}$. After this period, samples were centrifuged at $8.000 \mathrm{xg}$ at $4^{\circ} \mathrm{C}$ for $15 \mathrm{~min}$ and the supernatant was kept on ice until the PCR analyses.

For PCR, two pairs of primers were used: two universal primers, Uni-f: 5'-GCATATCAATAAGCGGAGGAAAAG-3' and Uni-r: 5'GGTCCGTGTTTCAAGACG-3'; and two C. dubliniensis-specific ones, DUBF Act-f: 5'GTATTTGTCGTTCCCCTTTC-3' and DUBR Act-r: 5'-GTGTTGTGTGCACTAACGTC-3'. The amplification was carried out in a $10 \mu \mathrm{L}$ final volume containing five picomoles of each, 5.0 $\mu \mathrm{L}$ PCR Master Mix (Promega, Madison, WI, USA), $3.2 \mu \mathrm{L}$ of ultra-pure water and $1 \mu \mathrm{L}$ of DNA template. Cycling conditions consisted of $3 \mathrm{~min}$ at $95^{\circ} \mathrm{C}$ followed by 30 cycles of $30 \mathrm{~s}$ at $95^{\circ} \mathrm{C}, 30 \mathrm{~s}$ at $58^{\circ} \mathrm{C}, 60 \mathrm{~s}$ at $72^{\circ} \mathrm{C}$, followed by $72^{\circ} \mathrm{C}$ for $10 \mathrm{~min}$ (Mastercycler, Eppendorf, Hamburg, Germany). In all reactions, C. albicans (ATCC 18804) and C. dubliniensis (NCPF 3108) were included as control. Amplification products were separated by electrophoresis through $2 \%(\mathrm{w} / \mathrm{v})$ 
agarose gels containing $25 \mu \mathrm{M}$ ethidium bromide (Sigma) and visualized on a UV transilluminator (Foto/UV 26; Bioresearch, Upland, CA, USA). A DNA "ladder" 100 pb (BRL; Gibco, Gaithrsbur, MD, USA) was used as molecular size standard. The gels were photographed for documentation (Kodak Photo Documentation System, DC290; Sigma).

\section{Statistical Analysis}

The heart transplant and control groups were compared for the presence (or absence) of Candida

Table 1- Number and percentage of individuals positive to Candida spp. in the oral cavity

\begin{tabular}{lccc}
\hline Groups & $\mathbf{n}$ & $\begin{array}{c}\text { Positive to } \\
\text { Candida spp. }\end{array}$ & $\begin{array}{c}\text { Negative to } \\
\text { Candida spp. }\end{array}$ \\
\hline Transplanted & 50 & $44(88 \%)^{*}$ & $6(12 \%)$ \\
Control & 50 & $28(56 \%)$ & $22(44 \%)$ \\
\hline
\end{tabular}

*Statistically different proportion in relation to the control (chi-square test, $p=0.001$ )

Table 2- Values of logarithm of colonies forming units per mililitre (log cfu/mL) in the studied groups

\begin{tabular}{lcccc}
\hline Groups & $\mathbf{n}$ & Median & $\mathbf{2 5 \%}$ & $\mathbf{7 5 \%}$ \\
\hline Transplanted & 50 & $5.66^{*}$ & 4.60 & 6.27 \\
Control & 50 & 5 & 0 & 5.55 \\
\hline
\end{tabular}

*Statistically significant difference in relation control group (Mann-Whitney, $p=0.005$ ) spp. by chi-square test in order to compare proportions.

Counts of yeasts were expressed as median values of logarithm of $\mathrm{cfu} / \mathrm{mL}$ and were statistically compared by Mann-Whitney's test, using the Minitab Statistical Software version 13.1 (Minitab Inc., State College, PA, USA). A significance level of $5 \%$ was set for all analyses.

\section{RESULTS}

The heart transplant group differed significantly $(p=0.001)$ from the control group for the presence of Candida spp. (Table 1 ). There was no statistically significant difference $(p=0.104)$ between the heart transplant and the control group for the proportion of the presence of $C$. albicans and others species.

Counts of yeasts (cfu/mL) in the transplanted group were significantly higher than in the control group $(p=0.005)$ (Table 2$)$.

C. albicans was the prevalent species in both groups of subjects. Among the transplanted patients, other isolated Candida species and Rhodotorula rubra was found. C. dubliniensis was not found in either of the groups. Larger counts of Candida species were found in the transplanted individuals in relation to the controls (Table 3 ). Four isolates of $R$. rubra were observed in the heart transplant group and 7 in the control group.

Associations of Candida species observed in the transplant recipients and controls are shown in Figure 1. More associations were observed in the transplantation group.

Table 3- Candida species isolated from the oral cavity of orthotopic cardiac transplantation patients and species isolated from the oral cavity of control individuals

\begin{tabular}{lcccc}
\hline \multirow{2}{*}{ Species } & \multicolumn{2}{c}{ Transplanted $(\mathbf{n}=\mathbf{6 7})$} & \multicolumn{2}{c}{ Control } \\
& $\mathbf{n}$ & $\mathbf{\%}$ & $\mathbf{n}$ & $\%$ \\
\hline C. albicans & 31 & 49.21 & 25 & 75.75 \\
C. glabrata & 9 & 14.28 & 3 & 9.1 \\
C. tropicalis & 7 & 11.11 & 2 & 6.06 \\
\hline C. krusei & 4 & 6.35 & 2 & 6.06 \\
C. parapsilosis & 4 & 6.35 & - & - \\
C. kefyr & 3 & 4.76 & - & - \\
C. lipolitica & 3 & 4.76 & - & - \\
C. guilliermondii & 1 & 1.59 & 1 & 3.03 \\
C.lusitaniae & 1 & 1.59 & - & - \\
Total & 63 & 100 & 33 & 100 \\
\hline
\end{tabular}




\begin{tabular}{|l|l|}
\hline Patients (n) & Associations of Candida Species \\
\hline & \\
\hline $\operatorname{Tx}(3)$ & C. albicans and C. parapsilosis \\
\hline $\operatorname{Tx}(2)$ & C. albicans and C. glabrata \\
\hline $\operatorname{Tx}(1)$ & C. albicans and C. kefyr \\
\hline $\operatorname{Tx}(1)$ & C. lusitaniae and C. glabrata \\
\hline $\operatorname{Tx}(1)$ & C. glabrata and C. krusei \\
\hline $\operatorname{Tx}(1)$ & C. albicans and C. guilliermondii \\
\hline $\operatorname{Tx}(1)$ & C. tropicalis and C. glabrata \\
\hline $\operatorname{Tx}(1)$ & C. albicans and C. krusei \\
\hline $\operatorname{Tx}(1)$ & C. albicans, C. tropicalis and C. kefyr \\
\hline $\operatorname{Tx}(1)$ & C. tropicalis, C. glabrata and C. lipolytica \\
\hline $\operatorname{Tx}(1)$ & C. albicans, C. tropicalis and C. krusei \\
\hline $\operatorname{Tx}(1)$ & C. albicans, C. tropicalis, C. krusei, C. glabrata and C. kefyr \\
\hline C (4) & C. albicans and C. glabrata \\
\hline C (1) & C. albicans and C. guilliermondii \\
\hline C (1) & C. albicans, C. tropicalis and C. krusei \\
\hline
\end{tabular}

Figure 1- Associations of Candida species isolated from the oral cavity of orthotopic heart transplant patients (Tx) and control individuals $(\mathrm{C})$

\section{DISCUSSION}

Transplantation is a treatment option for patients who have lost the normal function of their vital organs. Infectious complications are still among the major causes of morbidity and mortality during the period immediately following surgery ${ }^{6}$. Many authors ${ }^{1,9,11,21}$ agree that orthotopic heart transplant recipients show an increased prevalence of Candida yeasts, and this was confirmed by our results. According to Ruskin, et al.22(1992), the reduction of Candida species in the oropharyngeal area could potentially decrease the incidence of disseminated candidiasis that is often associated to death cases in immunosuppressed patients ${ }^{9}$. Dictar, et al. ${ }^{6}$ (2000), have reported that the mortality rate due to fungal infections in recipients of transplanted organs increased to $23.7 \%$, and that $75 \%$ of these cases occurred among heart transplant recipients. Candida spp. was responsible for $29 \%$ of such infections.

Many studies on immunosuppressed individuals ${ }^{1,6,9,11,15,18,29}$ point to $C$. albicans as the most prevalent species, which agrees with the results of the present study. A greater variety of species was found in the heart transplant group, and this results also agree with to previous findings in literature ${ }^{6,9,12}$. Furthermore, some authors have reported that, in addition to $C$. albicans, the species most frequently related to immunosuppression were C. tropicalis, C. glabrata ${ }^{6}$ and C. dubliniensis $3,11,18,27$. In the present study, $C$. albicans was the most prevalent species, followed by $C$. glabrata and $C$. tropicalis. C. dubliniensis was not found in either of the groups. In the control group, C. albicans, $R$. rubra and C. glabrata were the most prevalent species.
C. dubliniensis has many phenotypic features with $C$. albicans and the incidence of candidemia due $C$. dubliniensis is unknown mainly because of difficulty in distinguishing these two strains ${ }^{11,17,27}$. C. dubliniensis has been frequently associated to oral and systemic candidiasis in HIV-positive patients $4,19,23$ under chemotherapy and bone marrow transplant patients ${ }^{3,18}$, sometimes liable for death cases $^{28}$. Thus, it is very important to identify among the population of immunossuppressed patients those who have $C$. dubliniensis in their oral microbiota, and how big is this ratio ( $\mathrm{cfu} / \mathrm{mL}$ ).

Sabouraud dextrose agar with chloramphenicol and CHROMagar Candida were effective for Candida growth and, in both media, there was a higher prevalence of yeasts in the heart transplant group. This group presents species variation one third higher in relation to control group and these results were similar to those obtained for others authors ${ }^{6,9}$. Being chromogenic, CHROMagar allowed the identification of distinct colonies. This fact offered a better visualization of the various shapes of the yeasts.

There was higher isolation ( $\mathrm{cfu} / \mathrm{mL}$ ) on Sabouraud dextrose agar with chloramphenicol than in CHROMagar Candida for both groups. It is likely that this culture medium causes partial inhibition of some Candida isolates, which might be attributed to a higher selectivity of chromogenic components.

We used a reliable, simple, fast, and noninvasive method for studying the prevalence of Candida yeasts in oral cavity of patients subjected orthotopic heart transplantation. This method was also able to detect, among the studied population, those individuals with higher Candida $\mathrm{cfu} / \mathrm{mL}$ counts in saliva, and who might be more susceptible to candidiasis development. In such cases, the 
use of a proper antifungal prophylaxis may be indicated, aiming at avoiding the clinical oral manifestation of the disease, as well as its systemic dissemination ${ }^{14,20}$.

\section{CONCLUSION}

The number of Candida yeasts was significantly larger in the oral cavity of orthotopic heart transplant patients than in non-immunosuppressed subjects. There was higher prevalence of $C$. albicans in saliva isolates from both groups, but the proportion between this species and others was not statistically different. The heart transplantation group showed higher variation of Candida species in relation to the control group.

\section{ACKNOWLEDGEMENTS}

The authors would like to thank the State of São Paulo Research Foundation/FAPESP (04/10654-9 and 05/55135-1) for financial support.

\section{REFERENCES}

1- Allen CM, Saffer A, Meister RK, Beck FM, Bradway S. Comparison of a lesion-inducing isolate and a non-lesional isolate of Candida albicans in an immunosuppresion model of oral candidiasis. J Oral Pathol Med. 1994;23(3):133-9.

2- Back-Brito GN, Mota AJ, Vasconcellos TC, Querido SMR, Jorge AOC, Reis ASM, et al. Frequency of Candida spp. in the oral cavity of Brazilian HIV-positive patients and correlation with CD4 cell counts and viral load. Mycopathologia. 2009;167(2):81-7.

3- Brandt ME, Harrison LH, Pass M, Sofair AN, Huie S, Li R, et al. Candida dubliniensis fungemia: the first four cases in North America. Emerg Infect Dis. 2000;6(1):46-9.

4- Chavasco JK, Paula CR, Hirata MH, Aleva NA, Melo CE, Gambale $W$, et al. Molecular identification of Candida dubliniensis isolated from oral lesions of HIV-positive and HIV-negative patients in São Paulo, Brazil. Rev Inst Med Trop. 2006;48(1):21-6.

5- Dean DA, Burchard KW. Fungal infection in surgical patients. Am J Surg. 1996;171(3):374-82.

6- Dictar MO, Maiolo E, Alexander B, Jacob N, Verón MT. Mycoses in the transplanted patient. Med Mycol. 2000;38(1):251-8.

7- Donnely SM, Sullivan DJ, Shanley DB, Coleman DC. Phylogenetic analysis and rapid identification of Candida dubliniensis based on analysis of ACT1 intron and exon sequences. Microbiology. 1999;145:1871-82.

8- Gabler IG, Barbosa AC, Vilela RR, Lyon S, Rosa CA. Incidence and anatomic localization of oral candidiasis in patients with AIDS hospitalized in a public hospital in Belo Horizonte, MG, Brazil. J Appl Oral Sci. 2008;16(4):247-50.

9- Grossi P, Farina C, Fiocchi R, Gasperina DD. Prevalence and outcome of invasive fungal infections in 1963 thoracic organ transplant recipients: a multicenter retrospective study. Transplantation. 2000;70(1):112-6.

10- Holmberg C, Laine J, Jalanko H, Leijala M, Hoppu K. Conversion from cyclosporine to Neoral in pediatric recipients for kidney, liver, and heart transplantation. Transplant Proc. 1996;28(4):2262-3. 11- Jabra-Rizk MA, Falkler WA Jr, Merz WG, Baqui AA, Kelley JI, Meiller TF. Retrospective identification and characterization of Candida dubliniensis isolates among Candida albicans clinical laboratory isolates from immunodeficiency virus (HIV)-infected and non-HIV-infected individuals. J Clin Microbiol. 2000;38(6):2423-6.
12- Kaklamanos EG, Charalampidou M, Menexes G, Topitsoglou V, Kalfas S. Transient oral microflora in Greeks attending day centres for the ederly and residentes in homes for the ederly. Gerodontology. 2005;22(3):158-67.

13- Khocht A, Schneider LC. Periodontol management of gingival overgrowth in the heart transplant patient: a case report. J Periodontol. 1997;68(11):1140-6.

14- Knosalla C, Weng Y, Warnecke H, Hummel M, Yankah AC, Hofmeister J, et al. Mycotic aortic aneurysms after orthotopic heart transplantation: a three-case report and review of the literature. J Heart Lung Transplant. 1996;15(8):827-39.

15- Komiyama EY, Ribeiro PM, Junqueira JC, Koga-Ito CY, Jorge AOC. Prevalence of yeasts in the oral cavity of children treated with inhaled corticosteroids. Braz Oral Res. 2004;18(3):197-201. 16- Mähnss B, Sterhr, Schäfer W, Neuber K. Comparison of standard phenothypic assays with a PCR method to discriminated Candida albicans and Candida dubliniensis. Mycoses. 2005:48(1):55-61. 17- Mariano PLS, Milan EP, Matta DA, Colombo AL. Candida dubliniensis identification in Brazilian yeast stock collection. Mem Inst Oswaldo Cruz. 2003;98(4):533-8.

18- Meis JF, Ruhnke M, De Pauw BE, Odds FC, Siegert W, Verweij PE. Candida dubliniensis candidemia in patients with chemotherapyinduced neutropenia and bone marrow transplantation. Emerg Infect Dis. 1999;5(1):150-3.

19- Melo NR, Taguchi H, Culhari VVP, Sano A, Fukushima K, Miyaji M, et al. Candida dubliniensis in a Brazilian family with an HIV 1-infected child: identification, antifungal susceptibility, drug accumulation and sterol composition. Braz J Microbiol. $2006 ; 37(3): 237-43$.

20- Pinto TMS, Neves ACC, Leão MVP, Jorge AOC. Vinegar as an antimicrobial agent for control of Candida spp. in complete denture wearers. J Appl Oral Sci. 2008;16(6):385-90.

21- Reichenspurner $H$, Gamberg P, Nitschke M, Valantine H, Hunt $S$, Oyer $P E$, et al. Significant reduction in the number of fungal infections after lung, heart-lung, and heart transplantation using aerosolized amphotericin B prophylaxis. Transplant Proc. 1997;29(9):627-8.

22- Ruskin JD, Wood RP, Bailey MR, Whitmore CK, Shaw BW. Comparative trial of oral clotrimazole and nystatin for oropharyngeal candidiasis prophylaxis in orthotopic liver transplant patients. Oral Surg Oral Med Oral Pathol. 1992;74(5):567-71.

23- Salkin IF, Pruitt WR, Padhye AA, Sullivan D, Coleman D, Pincus $\mathrm{DH}$. Distinctive carbohydrate assimilation profiles used to identify the first clinical isolates of Candida dubliniensis recovered in the United States. J Clin Microbiol. 1998;36(5):1467.

24- Samaranayake LP, MacFarlane TW. Oral candidosis. London: Wright; 1990.

25- Sandven P. Laboratory identification and sensitivity testing of yeast isolates. Acta Odontol Scand. 1990;48:27-36.

26- Schorling SR, Kortinga HC, Froschb M, Mühlschlegel FA. The role of Candida dubliniensis in oral candidiasis in human immunodeficiency virus-infected individuals. Crit Rev Microbiol. $2000 ; 26(1): 59-68$.

27- Sullivan DJ, Moran GP, Coleman DC. Candida dubliniensis: ten years on. FEMS Microbiol Lett. 2005;253(1):9-17.

28- Tan AL, Wang GC, Chiu YW. Candida dubliniensis infection, Singapore. Emerg Infect Dis. 2002;8(4):445-6.

29- Tintelnot K, Haase G, Seibold M, Bergmann F, Staemmler $M$, Franz $T$, et al. Evaluation of phenotypic markers for selection and identification of Candida dubliniensis. J Clin Microbiol. 2000;38(4):1599-608.

30- Witzel AL, Silveira FR, Pires Mde F, Lotufo MA. Oral candidiasis in HIV+ patients under treatment with protease inhibitors. Braz Oral Res. 2008;22(4):371-7. 